\title{
Microlensing by natural wormholes: Theory and simulations
}

\author{
Margarita Safonova* \\ Department of Physics and Astrophysics, University of Delhi, New Delhi-7, India \\ Diego F. Torres ${ }^{\dagger}$ \\ Instituto Argentino de Radioastronomía, C.C.5, 1894 Villa Elisa, Buenos Aires, Argentina \\ and Department of Physics, Princeton University, New Jersey 08544 \\ Gustavo E. Romero \\ Instituto Argentino de Radioastronomía, C.C.5, 1894 Villa Elisa, Buenos Aires, Argentina
}

(Received 15 May 2001; published 29 November 2001)

\begin{abstract}
We provide an in depth study of the theoretical peculiarities that arise in effective negative mass lensing, both for the case of a point mass lens and source, and for extended source situations. We describe novel observational signatures arising in the case of a source lensed by a negative mass. We show that a negative mass lens produces total or partial eclipse of the source in the umbra region and also show that the usual Shapiro time delay is replaced with an equivalent time gain. We describe these features both theoretically, as well as through numerical simulations. We provide negative mass microlensing simulations for various intensity profiles and discuss the differences between them. The light curves for microlensing events are presented and contrasted with those due to lensing produced by normal matter. The presence or absence of these features in the observed microlensing events can shed light on the existence of natural wormholes in the Universe.
\end{abstract}

DOI: 10.1103/PhysRevD.65.023001

PACS number(s): 95.30.Sf, 04.20.Gz, 98.90.+s

\section{INTRODUCTION}

Wormhole solutions to the Einstein field equations have been extensively studied in the last decade (see Refs. [1,2] and references cited therein, as well as the book by Visser [3]). Wormholes basically represent bridges between otherwise separated regions of the space-time (see Fig. 1) and need a special kind of matter in order to exist. This matter, known as exotic, violates the energy conditions (EC), particularly the null (or averaged null) one [3-5].

To specify what we are referring to when talking about the energy conditions, we shall provide their pointwise form. Apart from the null (NEC), we have the weak (WEC), the strong (SEC), and the dominant (DEC) energy conditions. For a Friedmann-Robertson-Walker space-time and a diagonal stress-energy tensor $T_{\mu \nu}=(\rho,-p,-p,-p)$ with $\rho$ the energy density and $p$ the pressure of the fluid, they read

$$
\begin{aligned}
& \mathrm{NEC} \Leftrightarrow(\rho+p \geqslant 0), \\
& \mathrm{WEC} \Leftrightarrow(\rho \geqslant 0) \text { and }(\rho+p \geqslant 0), \\
& \mathrm{SEC} \Leftrightarrow(\rho+3 p \geqslant 0) \text { and }(\rho+p \geqslant 0), \\
& \mathrm{DEC} \Leftrightarrow(\rho \geqslant 0) \text { and }(\rho \pm p \geqslant 0) .
\end{aligned}
$$

The EC are, then, linear relationships between the energy density and the pressure of the matter generating the space-

\footnotetext{
*Email address: rita@iucaa.ernet.in

${ }^{\dagger}$ Email address: dtorres@venus.fisica.unlp.edu.ar; dtorres@princeton.edu

${ }^{\star}$ Email address: romero@irma.iar.unlp.edu.ar
}

time curvature. We can immediately see why the possible violations of the EC are so polemic. If NEC is violated, then WEC is also violated. Negative energy densities-and so negative masses-are thus physically admitted.

It is important to keep in mind that the EC of classical general relativity are only conjectures. They are widely used to prove theorems concerning singularities and black hole thermodynamics, such as the area increase theorem, the topological censorship theorem, and the singularity theorem of stellar collapse [3]. However, the EC lack a rigorous proof from fundamental principles. Moreover, several situations in which they are violated are known, perhaps the most quoted being the Casimir effect, see Refs. [2,6]. Typically, observed violations are produced by small quantum systems, resulting of the order of $\hbar$. It is currently far from clear whether there could be macroscopic quantities of such an exotic, e.g. WEC-violating, matter. If it does exist, macroscopic negative masses could be part of the ontology of the Universe.

In fact, the possible existence of negative gravitational
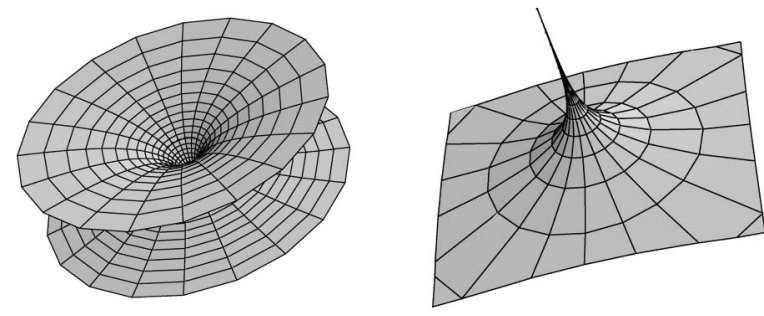

FIG. 1. Left: embedding diagram for a wormhole. Two mouths, joined by a tunnel, can connect regions otherwise very much separated (here the normal space should fold as a sheet of paper whereas the wormhole would be a tunnel from one side of the sheet to the other). Right: embedding diagram for a black hole. The singularity here is represented as a pinch off of the wormhole tunnel. 
masses is being investigated, at least, since the end of the nineteenth century [7]. From a Newtonian point of view, we can differentiate four possible situations [8]: (i) all masses are positive, (ii) inertial mass is positive and gravitational mass is negative, (iii) inertial mass is negative and the gravitational mass is positive, and (iv) all masses are negative. Most of the nineteenth century literature on negative masses is devoted to case (ii). From a relativistic point of view, however, the situation is quite different; the equivalence principle requires that gravitational and inertial masses cannot be considered distinct any longer. Test particles move along geodesic lines in accordance with the initial conditions, independently of the fact that their energy density is positive or negative. Negative energy densities or negative masses, then, are repulsive not only for ordinary matter but for exotic matter as well. This might conceivably pose a stability problem for large amounts of exotic material, requiring large values of tension in order to keep the equilibrium configuration [1]. In Ref. [3], Visser discusses some conditions to be satisfied for a dynamic wormhole in order to be stable against explosion. In more complicated astrophysical systems, stability could be achieved through electromagnetic forces.

Since wormholes have to violate the null energy condition in order to exist, the hypothesis underlying the positive mass theorem no longer applies, and there is nothing in principle that can prevent the occurrence of a negative total mass. Indeed, this is just a way of saying that we need to have some negative mass near the throat to keep the wormholes open [4]. Examples of wormhole solutions, both with positive and negative mass, quickly came out after the pioneering work by Morris and Thorne [1] (see [3] for a review). Visser, for instance, suggested a particular class of solutions, lacking spherical symmetry configurations in which there is a flatspace wormhole framed by struts of an exotic material variant of the cosmic strings solution [9]. These kinds of wormholes and many others, including the simplest one known as an absurdly benign wormhole [1], would have negative mass density. Typically, near the throat the following relationship holds:

$$
\text { mass at throat }=-\frac{\operatorname{radius} \times c^{2}}{G} .
$$

Then, for a radius equal to $1 \mathrm{~m}$ the mass of the mouth will be equal to -1 Jupiter mass. The total mass seen at infinity, the imprint of the wormhole on the whole space-time, will depend on the details of the model, such as the neighboring matter, and can be positive, negative or zero according to the specific case. Whether or not mass separation is possible is not clear yet.

Some speculations have been made about how inflation can be responsible to enlarge a microscopic wormholebelieved to exist in the Planck foam-out to microscopic dimensions [10]. This mechanism could result in a population of natural wormholes.

Recently, Mann [11] has demonstrated that, under certain circumstances, regions of negative energy density can undergo gravitational collapse into a black hole. The resulting exterior black hole space-times necessarily have negative mass and non-trivial topology. Mann modeled the negative masses with a stress energy tensor for a cloud of freely falling dust, given by $T_{\mu \nu}=-|\rho| u_{\mu} u_{\nu}$, where the energy density $\rho<0$ and $u_{\nu}$ is the associated four velocity. He required that the metric exterior to the dust cloud must (a) have a negative Arnowitt-Deser-Misner- (ADM-) type mass parameter and (b) have (in its maximal extension) all of its singularities cloaked by event horizons. A complete understanding of negative mass black holes yet remains to be attained, as well as of the precise astrophysical limits on the negative mass that these objects could give.

Clearly, no better way than devising observational tests for deciding on negative mass existence is available. For instance, if natural wormholes with negative masses actually exist in the universe (e.g. if the original topology after the big-bang was multiply connected), then there could be some observable electromagnetic signatures that might lead to their identification. The approach of the present work points in this direction; we do not know whether there exists an astrophysical population of objects with negative mass, but if they indeed exist, then we will be providing the specific observational signatures that we can expect.

A specific comment is now in order. Since the primary motivation for our theoretical work is observational, it is appropriate to set the experimental context more explicitly. In this paper, as in the work by Cramer et al. [13], we take wormholes as a useful and appealing theoretical scenario for the appearance of negative masses, but it applies, mutatis mutandis, to any possible object possessing a negative mass. Discovery of such objects will not prove the existence of wormholes for sure, although it will certainly enhance the possibilities for wormholes to exist. Equivalently, if after extensive searches, we disprove the existence of negative masses, wormholes with positive total mass can still be part of the Universe, and they would need to be unveiled by alternative techniques.

The idea that wormholes can act as gravitational lenses and induce a microlensing signature on a background source was first suggested by Kim and Cho [12]. Unfortunately, their geometry was that of a perfect alignment of a source, both wormhole's mouths and an observer, which is, on a common sense ground, quite unlikely. They also considered both mouths to be of positive mass. Cramer et al. [13] carried out more detailed analysis of negative mass wormholes and considered the effect they can produce on background point sources, at non-cosmological distances. The generalization to a cosmological scenario was carried out by Torres et al. [14], although lensing of point sources was still used. As far as we are aware, the first and only bound on the possible existence of negative masses, imposed using astrophysical databases, was given by Torres et al. [14]. These authors showed that the effective gravitational repulsion of light rays creates two bursts, which are individually asymmetric under time reversal, although mirror images of each other. Recently, Anchordoqui et al. [15] searched in existent gamma-ray bursts databases for signatures of wormhole microlensing. Although they detected some interesting candidates, no conclusive results could be obtained. Peculiarly 
asymmetric gamma-ray bursts [16], although highly uncommon, could probably be explained by more conventional hypotheses, such as precessing fireballs (see, for instance, Ref. [17]).

The case of macrolensing was recently analyzed by us as well, showing that if large concentrations of localized negative masses do exist, we should be able to see distinctive effects upon a deep background [18]. All in all, the possible existence of wormholes or of any kind of negative masses in the Universe cannot yet be discarded. In order to confirm or to deny the existence of such masses, we need to develop a strong theoretical framework and to provide a clear test through observations.

In this work we revisit the microlensing by natural wormholes of stellar and sub-stellar masses. We provide an indepth study of the theoretical peculiarities that arise in negative mass microlensing, both for a point mass lens and source, and for extended source situations. For the first time, we present negative mass microlensing simulations, showing the resulting shapes of the images, the intensity profiles, the time gain function, the radial and tangential magnifications, and other features. In this regard, this work extends and deepens previous papers in several ways, and gives a complete description from where to analyze, from a computational and quantitative point of view, observational predictions, as the ones presented for chromaticity in Ref. [19]. Although we will deal with a Schwarzschild lens, our treatment in the weak field approximation will be useful to study lensing by any compact object which, seen from a large distance, appears basically as a point lens.

The article is divided as follows. In Sec. II we introduce the basic theoretical framework of gravitational lensing produced by a generic negative mass, assumed-as just explained - to be a wormhole. Note that we never consider light going through the wormhole mouths, but being deflected in the neighborhood. We introduce the effective refractive index, magnification results, the time gain function, and other features, in several subsections of Sec. II. Section III presents microlensing simulations, showing the form and position of the produced images. In Sec. IV, we treat the extended source case, also using a numerical code and taking into account different source profiles. We finally give some concluding remarks.

\section{LENSING BY A POINT OF NEGATIVE MASS}

In this paper we consider lensing only by a point negative mass lens, and thus we can use all the assumptions concurrent with the treatment of the Schwarzschild lens:

Geometrical optics approximation - the scale over which the gravitational field changes is much larger than the wavelength of the light being deflected.

Small-angle approximation - the total deflection angle is small. The typical bending angles involved in gravitational lensing of cosmological interest are less than $<1^{\prime}$; therefore we describe the lens optics in the paraxial approximation.

Geometrically-thin lens approximation-the maximum deviation of the ray is small compared to the length scale on which the gravitational field changes. Although the scattering takes place continuously over the trajectory of the photon, appreciable bending occurs only within a distance of the order of the impact parameter.

We begin the discussion of gravitational lensing by defining two planes, the source and the lens plane. These planes, described by Cartesian coordinate systems $\left(\xi_{1}, \xi_{2}\right)$ and $\left(\eta_{1}, \eta_{2}\right)$, respectively, pass through the source and deflecting mass and are perpendicular to the optical axis (the straight line extended from the source plane through the deflecting mass to the observer). Since the components of the image position and the source positions are much smaller in comparison to the distances to lens and source planes, we can write the coordinates in terms of the observed angles. Therefore, the image coordinates can be written as $\left(\theta_{1}, \theta_{2}\right)$ and those of the source as $\left(\beta_{1}, \beta_{2}\right)$.

\section{A. Effective refractive index of the gravitational field of a negative mass and the deflection angle}

The "Newtonian" potential of a negative point mass lens is given by

$$
\Phi(\xi, z)=\frac{G|M|}{\left(b^{2}+z^{2}\right)^{1 / 2}},
$$

where $b$ is the impact parameter of the unperturbed light ray and $z$ is the distance along the unperturbed light ray from the point of closest approach. We have used the term Newtonian in quotation marks since it is, in principle, different from the usual one. Here the potential is positive defined and approaching zero at infinity [3]. In view of the assumptions stated above, we can describe light propagation close to the lens in a locally Minkowskian space-time perturbed by the positive gravitational potential of the lens to first postNewtonian order. In this weak field limit, we describe the metric of the negative mass body in orthonormal coordinates $x^{0}=c t, \mathbf{x}=\left(x^{i}\right)$ by

$$
d s^{2} \approx\left(1+\frac{2 \Phi}{c^{2}}\right) c^{2} d t^{2}-\left(1-\frac{2 \Phi}{c^{2}}\right) d l^{2},
$$

where $d l=|\mathbf{x}|$ denotes Euclidean arc length. The effect of the space-time curvature on the propagation of light can be expressed in terms of an effective index of refraction $n_{\text {eff }}$ [20], which is given by

$$
n_{\mathrm{eff}}=1-\frac{2}{c^{2}} \Phi
$$

Thus, the effective speed of light in the field of a negative mass is

$$
v_{\mathrm{eff}}=c / n_{\mathrm{eff}} \approx c+\frac{2}{c} \Phi .
$$

Because of the increase in the effective speed of light in the gravitational field of a negative mass, light rays would arrive faster than those following a similar path in vacuum. This leads to a very interesting effect when compared with the 
propagation of a light signal in the gravitational field of a positive mass. In that case, light rays are delayed relative to propagation in vacuum - the well known Shapiro time delay. In the case of a negative mass lensing, this effect is replaced by a new one, which we shall call time gain. We will describe this effect in more detail in the following subsections.

Defining the deflection angle as the difference of the initial and final ray direction

$$
\boldsymbol{\alpha}:=\hat{\mathbf{e}}_{\mathrm{in}}-\hat{\mathbf{e}}_{\mathrm{out}},
$$

where $\hat{\mathbf{e}}:=d \mathbf{x} / d l$ is the unit tangent vector of a ray $\mathbf{x}(l)$, we obtain the deflection angle as the integral along the light path of the gradient of the gravitational potential

$$
\boldsymbol{\alpha}=\frac{2}{c^{2}} \int \boldsymbol{\nabla}_{\perp} \Phi d l
$$

where $\nabla_{\perp} \Phi$ denotes the projection of $\nabla \Phi$ onto the plane orthogonal to the direction $\hat{\mathbf{e}}$ of the ray. We find

$$
\boldsymbol{\nabla}_{\perp} \Phi(b, z)=-\frac{G|M| \mathbf{b}}{\left(b^{2}+z^{2}\right)^{3 / 2}} .
$$

Equation (7) then yields the deflection angle

$$
\boldsymbol{\alpha}=-\frac{4 G|M| \mathbf{b}}{c^{2} b^{2}} .
$$

It is interesting to point out that in the case of the negative mass lensing, the term "deflection" has its rightful meaning - the light is deflected away from the mass, unlike in the positive mass lensing, where it is bent towards the mass.

\section{B. Lensing geometry and lens equation}

In Fig. 2 we present the lensing geometry for a point-like negative mass. From this figure and the definition of the deflection angle [Eq. (6)], we can obtain the relation between the positions of the source and the image. We only need to relate the radial distance of the source and the image from the center, since due to circular symmetry, the azimuthal angle $\varphi$ is not affected by lensing. This gives

$$
(\boldsymbol{\beta}-\boldsymbol{\theta}) D_{\mathrm{s}}=-\boldsymbol{\alpha} D_{\mathrm{ls}}
$$

or

$$
\boldsymbol{\beta}=\boldsymbol{\theta}-\frac{D_{\mathrm{ls}}}{D_{\mathrm{s}}} \boldsymbol{\alpha}
$$

With the deflection angle [Eq. (9)], we can write the lens equation as

$$
\beta=\theta+\frac{4 G|M|}{c^{2} \xi} \frac{D_{l s}}{D_{s}}=\theta+\frac{4 G|M|}{c^{2}} \frac{D_{l s}}{D_{s} D_{l}} \frac{1}{\theta} .
$$

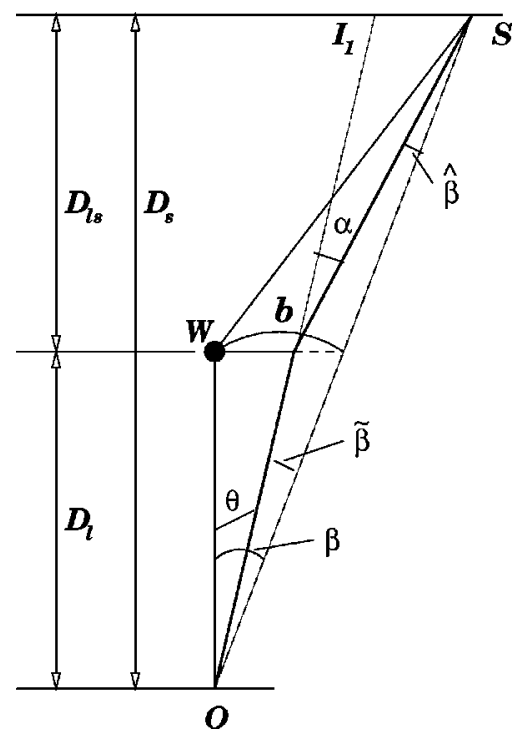

FIG. 2. Lensing geometry of a negative mass. $O$ is the observer, $S$ is the source, $W$ is the negative mass lens, $I_{1}$ is one of the images. $\beta$ is the angle between the source and the lens-position of the source, $\theta$ is the angle between the source and the image-position of the image, and $\alpha$ is the deflection angle. $b$ is the impact parameter and $D_{l}, D_{s}$ and $D_{l s}$ are angular diameter distances. Other quantities are auxiliary.

\section{Einstein radius and the formation of images}

A natural angular scale in this problem is given by the quantity

$$
\theta_{\mathrm{E}}^{2}=\frac{4 G|M|}{c^{2}} \frac{D_{\mathrm{ls}}}{D_{\mathrm{s}} D_{1}}
$$

which is called the Einstein angle. In the case of a positive point mass lens, this corresponds to the angle at which the Einstein ring is formed, happening when source, lens and observer are perfectly aligned. As we will see later in this section, this does not happen if the mass of the lens is negative. There are other differences as well. A typical angular separation of images is of order $2 \theta_{\mathrm{E}}$ for a positive mass lens. Sources which are closer than about $\theta_{\mathrm{E}}$ to the optical axis are significantly magnified, whereas sources which are located well outside the Einstein ring are magnified very little. All this is different with a negative mass lens, but nonetheless, the Einstein angle remains a useful scale for the description of the various regimes in the present case and, therefore, we shall use the same nomenclature for its definition.

The Einstein angle corresponds to the Einstein radius in the linear scale (in the lens plane):

$$
R_{\mathrm{E}}=\theta_{\mathrm{E}} D_{1}=\sqrt{\frac{4 G M}{c^{2}} \frac{D_{\mathrm{ls}} D_{1}}{D_{\mathrm{s}}}}
$$

In terms of the Einstein angle the lens equation takes the form 


$$
\beta=\theta+\frac{\theta_{\mathrm{E}}^{2}}{\theta}
$$

which can be solved to obtain two solutions for the image position $\theta$ :

$$
\theta_{1,2}=\frac{1}{2}\left(\beta \pm \sqrt{\beta^{2}-4 \theta_{\mathrm{E}}^{2}}\right) .
$$

Unlike in the lensing due to positive masses, we find that there are three distinct regimes here and, thus, can classify the lensing phenomenon as follows:

(I) $\beta<2 \theta_{\mathrm{E}}$-There is no real solution for the lens equation. It means that there are no images when the source is inside twice the Einstein angle.

(II) $\beta>2 \theta_{\mathrm{E}}$-There are two solutions, corresponding to two images both on the same side of the lens and between the source and the lens. One is always inside the Einstein angle, the other is always outside it.

(III) $\beta=2 \theta_{\mathrm{E}}$-This is a degenerate case, $\theta_{1,2}=\theta_{\mathrm{E}}$; two images merge at the Einstein angular radius, forming the radial arc (see Sec. II E).

We also obtain two important scales: one is the Einstein angle $\left(\theta_{\mathrm{E}}\right)$ - the angular radius of the radial critical curve, the other is twice the Einstein angle $\left(2 \theta_{\mathrm{E}}\right)$-the angular radius of the caustic. Thus, we have two images, one is always inside the $\theta_{\mathrm{E}}$, one is always outside; as a source approaches the caustic $\left(2 \theta_{\mathrm{E}}\right.$ ) from the positive side, two images coming closer and closer together, and nearer the critical curve, thereby brightening. When the source crosses the caustic, the two images merge on the critical curve $\left(\theta_{\mathrm{E}}\right)$ and disappear.

\section{Time gain and time-offset function}

Following [21], we define a scalar potential, $\psi(\boldsymbol{\theta})$, which is the appropriately scaled projected Newtonian potential of the lens,

$$
\psi(\boldsymbol{\theta})=\frac{D_{\mathrm{ls}}}{D_{1} D_{\mathrm{s}}} \frac{2}{c^{2}} \int \Phi\left(D_{1} \boldsymbol{\theta}, z\right) d z
$$

For a negative point mass lens it is

$$
\psi(\boldsymbol{\theta})=\frac{D_{1 \mathrm{~s}}}{D_{1} D_{\mathrm{s}}} \frac{4 G|M|}{c^{2}} \ln |\theta|
$$

The derivative of $\psi$ with respect to $\boldsymbol{\theta}$ is the deflection angle

$$
\boldsymbol{\nabla}_{\theta} \psi=D_{l} \boldsymbol{\nabla}_{b} \psi=\frac{2}{c^{2}} \frac{D_{l s}}{D_{s}} \int \boldsymbol{\nabla}_{\perp} \Phi d z=\boldsymbol{\alpha}
$$

Thus, the deflection angle is the gradient of $\psi$-the deflection potential

$$
\boldsymbol{\alpha}(\boldsymbol{\theta})=\nabla_{\theta} \psi
$$

From this fact and the lens equation (11) we obtain

$$
(\boldsymbol{\theta}-\boldsymbol{\beta})+\nabla_{\theta} \psi(\boldsymbol{\theta})=0 \text {. }
$$

This equation can be written as a gradient,

$$
\boldsymbol{\nabla}_{\theta}\left[\frac{1}{2}(\boldsymbol{\theta}-\boldsymbol{\beta})^{2}+\psi(\boldsymbol{\theta})\right]=0 .
$$

If we compare this equation with that for the Fermat's principle [21]

$$
\nabla_{\theta} t(\boldsymbol{\theta})=0,
$$

we see that we can define the time-offset function (opposite to time-delay function in the case of positive mass lens) as

$$
t(\boldsymbol{\theta})=\frac{\left(1+z_{1}\right)}{c} \frac{D_{1} D_{\mathrm{s}}}{D_{\mathrm{ls}}}\left[\frac{1}{2}(\boldsymbol{\theta}-\boldsymbol{\beta})^{2}+\psi(\boldsymbol{\theta})\right]=t_{\mathrm{geom}}+\tilde{t}_{\mathrm{pot}},
$$

where $t_{\text {geom }}$ is the geometrical time delay due to the extra path length of the deflected light ray relative to the unperturbed one. It remains the same as in the positive caseincrease of light-travel-time relative to an unbent ray. The coefficient in front of the square brackets ensures that the quantity corresponds to the time offset as measured by the observer. The second term $\tilde{t}_{\text {pot }}$ is the time gain a ray experiences as it traverses the deflection potential $\psi(\boldsymbol{\theta})$, with an extra factor $\left(1+z_{l}\right)$ for the cosmological "redshifting." Thus, cosmological geometrical time delay is

$$
t_{\text {geom }}=\frac{\left(1+z_{1}\right)}{c} \frac{D_{1} D_{\mathrm{s}}}{D_{\text {ls }}} \frac{1}{2}(\boldsymbol{\theta}-\boldsymbol{\beta})^{2},
$$

and cosmological potential time gain is

$$
\tilde{t}_{\text {pot }}=\frac{\left(1+z_{1}\right)}{c} \frac{D_{1} D_{\mathrm{s}}}{D_{\text {ls }}} \psi(\boldsymbol{\theta}) .
$$

In Fig. 3 we show the time delay and time gain functions. The top panel shows $t_{\text {geom }}$ for a slightly offset source. The curve is a parabola centered on the position of the source $\beta$. The central panel displays $\tilde{\mathrm{t}}_{\text {pot }}$ for a point negative mass lens. This curve is centered on the lens. The bottom panel shows the total time-offset. Images are located at the stationary points of $t_{\text {total }}$. Here we see two extrema-maximum and minimum - on the same side (right) from the optical axis (marked by dots).

We can find the time difference between the two images, $\theta_{1}$ and $\theta_{2}$, that is, if a source has intrinsic variability, it will appear in the two images at an interval

$$
\Delta t_{12}=\frac{r_{\mathrm{s}}}{c}\left(1+z_{1}\right)\left(\nu^{1 / 2}-\nu^{-1 / 2}-\ln \nu\right),
$$

where by $\nu$ we denoted the ratio of absolute values of magnifications of images,

$$
\frac{\mu_{1}}{\mu_{2}}=\left[\frac{\sqrt{u^{2}-4}+u}{\sqrt{u^{2}-4}-u}\right]^{2},
$$

and $u$ is the scaled angular position of the source $u=\beta / \theta_{\mathrm{E}}$ and $r_{\mathrm{s}}$ is the Schwarzschild radius of the lens. 


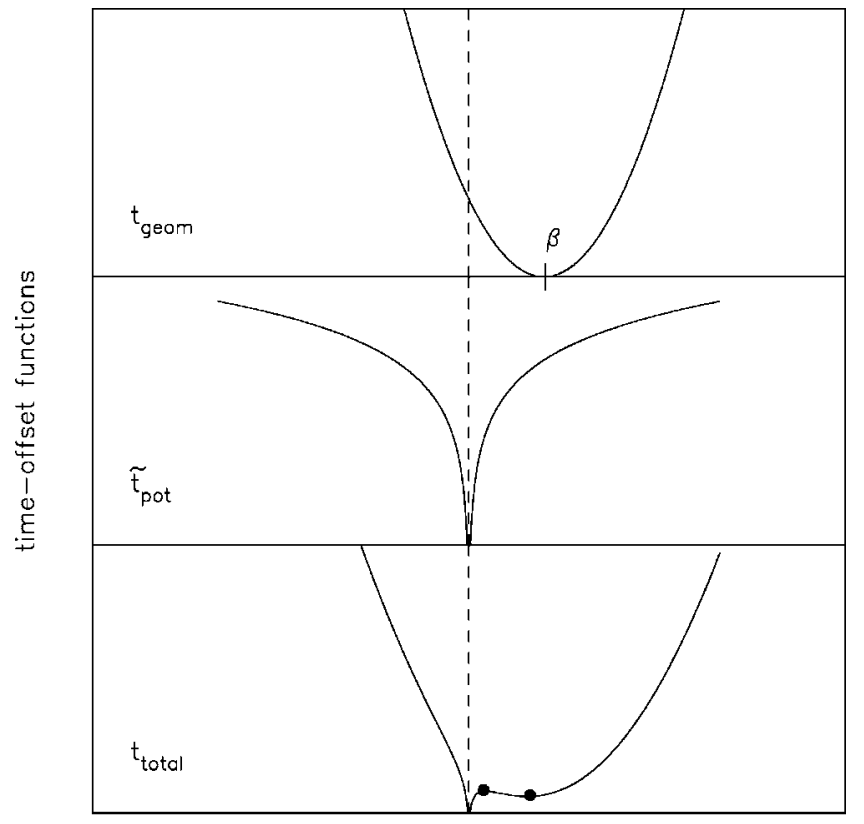

angular position

FIG. 3. Geometric time delay, gravitational time gain and total time offset produced by a point negative mass lens for a source that is slightly off the optical axis.

\section{E. Magnifications}

Light deflection not only changes the direction but also the cross section of a bundle of rays. For an infinitesimally small source, the ratio between the solid angles gives the flux amplification due to lensing

$$
|\mu|=\frac{d \omega_{i}}{d \omega_{s}} .
$$

For an infinitesimal source at angular position $\boldsymbol{\beta}$ and image at angular position $\boldsymbol{\theta}$, the relation between the two solid angles is determined by the area distortion, given in turn by the determinant of the Jacobian matrix $\mathcal{A}$ of the lens mapping $\boldsymbol{\theta} \mapsto \boldsymbol{\beta}$

$$
\mathcal{A} \equiv \frac{\partial \boldsymbol{\beta}}{\partial \boldsymbol{\theta}} .
$$

For a point mass lens magnification is given by

$$
\mu^{-1}=\left|\frac{\beta}{\theta} \frac{d \beta}{d \theta}\right| .
$$

The image is thus magnified or demagnified by a factor of $|\mu|$. If a source is mapped into several images, the total amplification is given by the sum of the individual image magnifications. From the lens equation (15), we find

$$
\frac{\beta}{\theta}=\frac{\theta^{2}+\theta_{\mathrm{E}}^{2}}{\theta^{2}} ; \quad \frac{d \beta}{d \theta}=\frac{\theta^{2}-\theta_{\mathrm{E}}^{2}}{\theta^{2}} .
$$

Thus,

$$
\mu_{1,2}^{-1}=\left|1-\frac{\theta_{\mathrm{E}}^{4}}{\theta_{1,2}^{4}}\right|,
$$

and using $u$ from Eq. (28), we find the total magnification (Fig. 4, bottom panel, continuous curve) as

$$
\mu_{\mathrm{tot}}=\left|\mu_{1}\right|+\left|\mu_{2}\right|=\frac{u^{2}-2}{u \sqrt{u^{2}-4}} .
$$

The tangential and radial critical curves follow from the singularities in

$$
\mu_{\tan }=\left|\frac{\beta}{\theta}\right|^{-1}=\frac{\theta^{2}}{\theta^{2}+\theta_{\mathrm{E}}^{2}}
$$

and

$$
\mu_{\mathrm{rad}}=\left|\frac{d \beta}{d \theta}\right|^{-1}=\frac{\theta^{2}}{\theta^{2}-\theta_{\mathrm{E}}^{2}} .
$$

$\mu_{\text {rad }}$ diverges when $\theta=\theta_{\mathrm{E}}$-angular radius of the radial critical curve. $\mu_{\tan }$ always remains finite, which means that there are no tangential critical curves-no tangential arcs can be formed by the negative point mass lens. In Fig. 4 we show the magnification curves (radial, tangential and total) for both positive (upper panel) and negative mass lenses (bottom panel). The difference can be seen as follows-in the upper panel there is no singularity in the radial curve (no radial arcs are formed by the positive mass lens), whereas in the bottom panel we see that the curve for the radial magnification experiences a singularity.

\section{MICROLENSING}

When the angular separation between the images $\Delta \theta$

$$
\Delta \theta=\sqrt{\beta^{2}-4 \theta_{\mathrm{E}}^{2}}
$$

is of the order of milliarcsecs, we cannot resolve the two images with existing telescopes and we can only observe the lensing effect through their combined light intensity. This effect is called microlensing. Both the lens and the source are moving with respect to each other (as well as the observer). Thus, images change their position and brightness. Of particular interest are sudden changes in luminosity, which occur when a compact source crosses a critical curve. For the positive mass lensing the situation is quite simple (Fig. 5) (for a review on the positive mass microlensing and its applications, see [27]).

For a negative mass lens the situation is different. We define a dimensionless minimum impact parameter $B_{0}$, expressed in terms of the Einstein radius, as the shortest distance between the path line of the source and the lens. For three different values of $B_{0}$ we have three different lensing configurations shown in Figs. 6, 7, and 8. Note the large difference in the shapes of the images for these three re- 

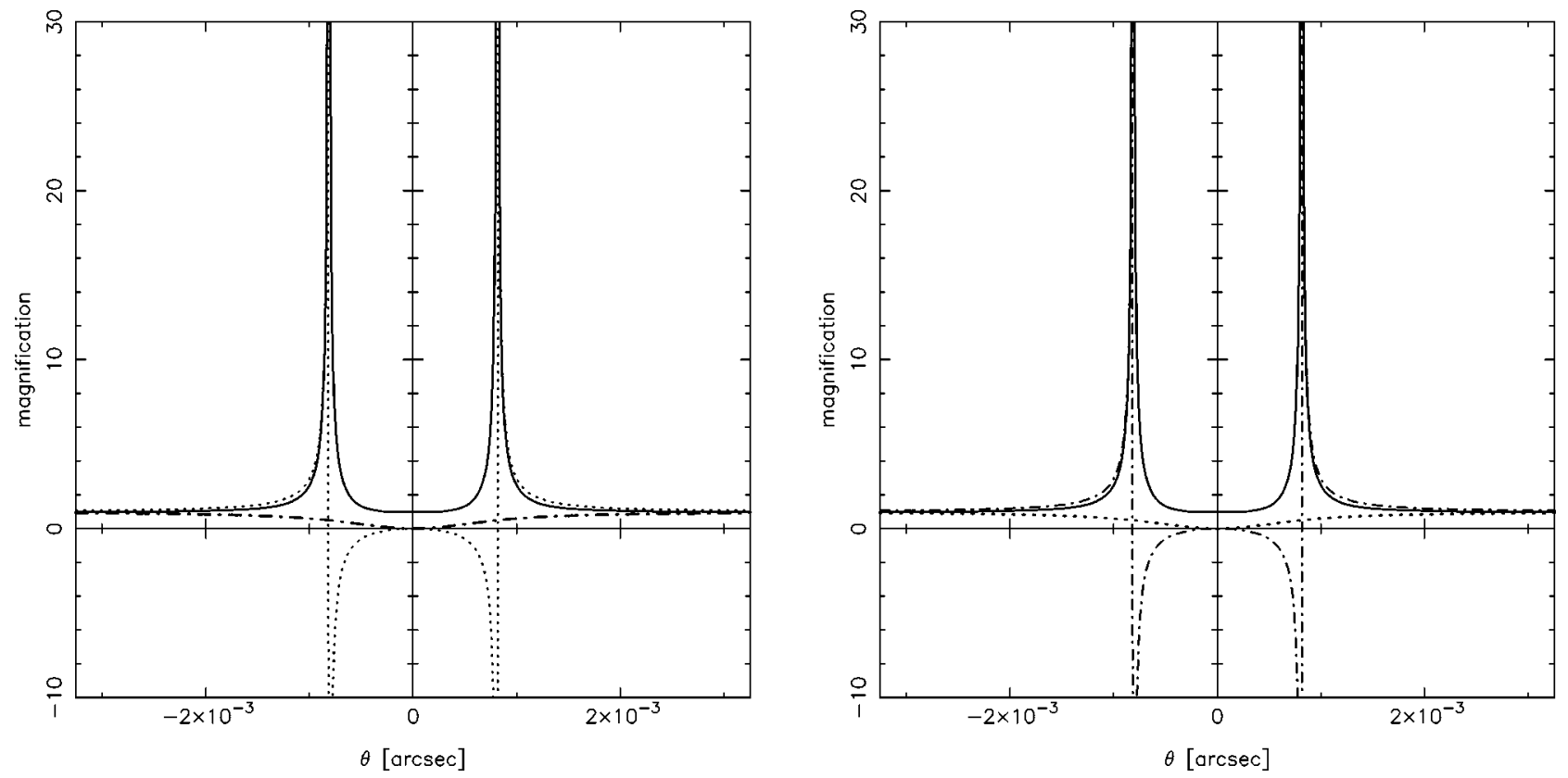

FIG. 4. The magnifications, tangential $\mu_{\mathrm{tan}}$ (dotted lines), radial $\mu_{\text {rad }}$ (dash-dotted lines), and total $\mu$ (continuous curves), are plotted as functions of the image position $\theta$ for two cases. In the upper panel for the positive mass, in the bottom panel for the negative mass. The singularities of $\mu_{\tan }$ and $\mu_{\text {rad }}$ give the positions of the tangential and radial critical curves, respectively. In the upper panel the singularity is in the tangential critical curve, in the bottom panel, instead, in the radial critical curve. Here $|M|=1 M_{\odot}, D_{\mathrm{s}}=0.05 \mathrm{Mpc}$ and $D_{1}$ $=0.01 \mathrm{Mpc}$. Angles are in arcseconds.

gimes. In Fig. 9 we show the case of a minimum impact parameter equal to zero, $B_{0}=0$, that is, the path of the source passes through the lens.

It can be assumed without the loss of generality that the observer and the lens are motionless and the source moves in

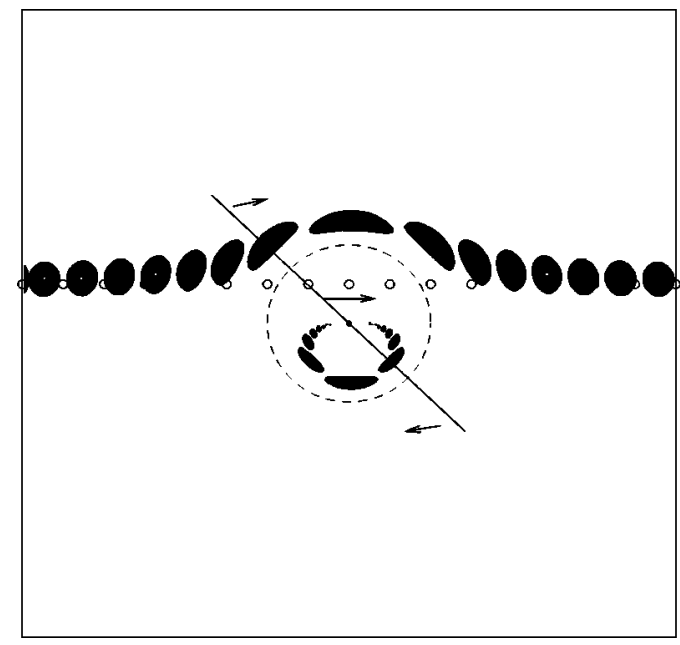

FIG. 5. Schematic representation of the geometry of the positive mass lensing due to the motion of the source, lens and the observer (in this case we can consider only the motion of the source in the plane perpendicular to the optical axis). The lens is indicated with a dot at the center of the Einstein ring, which is marked with a dashed line. The positions of the source center are shown with a series of small open circles. The locations and the shapes of the two images are shown with a series of dark ellipses. At any instant, the two images, the source and the lens are all on a single line, as shown in the figure for one particular instant. the plane perpendicular to the line of sight (therefore, changing its position in the source plane). We adopt the treatment given in [22] for the velocity $V$, and consider effective transverse velocity of the source relative to the critical curve (see Appendix A). We define the time scale of the microlensing event as the time it takes the source to move across the Einstein radius, projected onto the source plane, $\xi_{0}=\theta_{\mathrm{E}} D_{\mathrm{s}}$,

$$
t_{\mathrm{v}}=\frac{\xi_{0}}{V}
$$

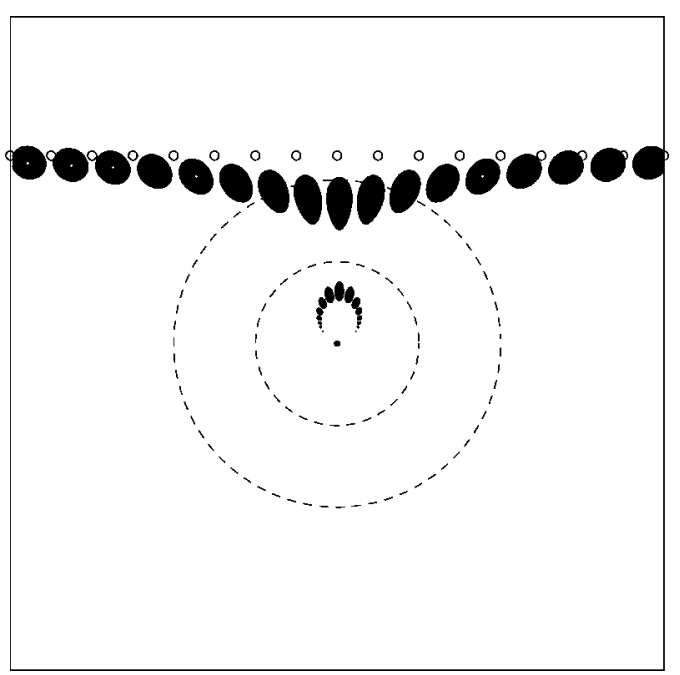

FIG. 6. True motion of the source and apparent motion of the images for $B_{0}>2$. The inner dashed circle is the Einstein ring, the outer dashed circle is twice the Einstein ring. The rest is as in Fig. 5. 


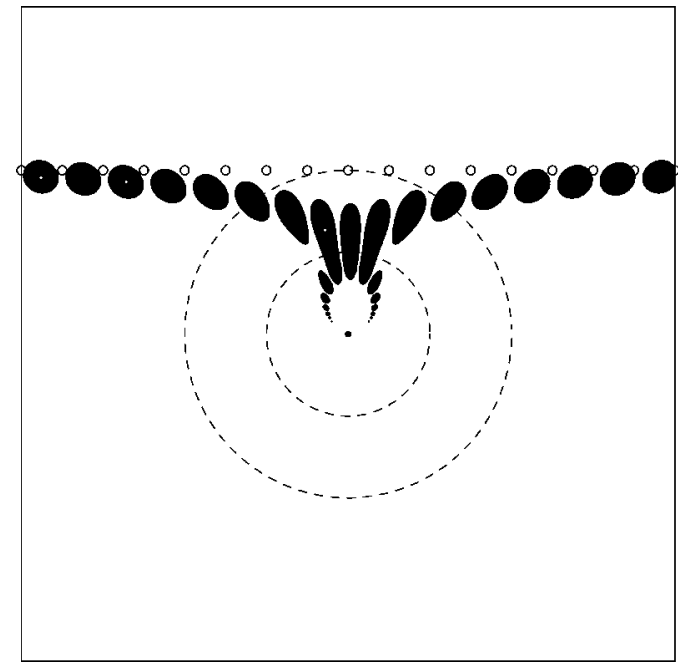

FIG. 7. True motion of the source and apparent motion of the images for $B_{0}=2$. The inner dashed circle is the Einstein ring, the outer dashed circle is twice the Einstein ring. The rest is as in Fig. 5.

Angle $\beta$ changes with time according to

$$
\beta(t)=\sqrt{\left(\frac{V t}{D_{\mathrm{s}}}\right)^{2}+\beta_{0}^{2}} .
$$

Here the moment $t=0$ corresponds to the smallest angular distance $\beta_{0}$ between the lens and the source. Normalizing Eq. (39) to $\theta_{E}$,

$$
u(t)=\sqrt{\left(\frac{V t}{\theta_{\mathrm{E}} D_{\mathrm{s}}}\right)^{2}+\left(\frac{\beta_{0}}{\theta_{\mathrm{E}}}\right)^{2}},
$$

where dimensionless impact parameter $u$ is defined in Eq. (28). Including the time scale $t_{v}$ and defining

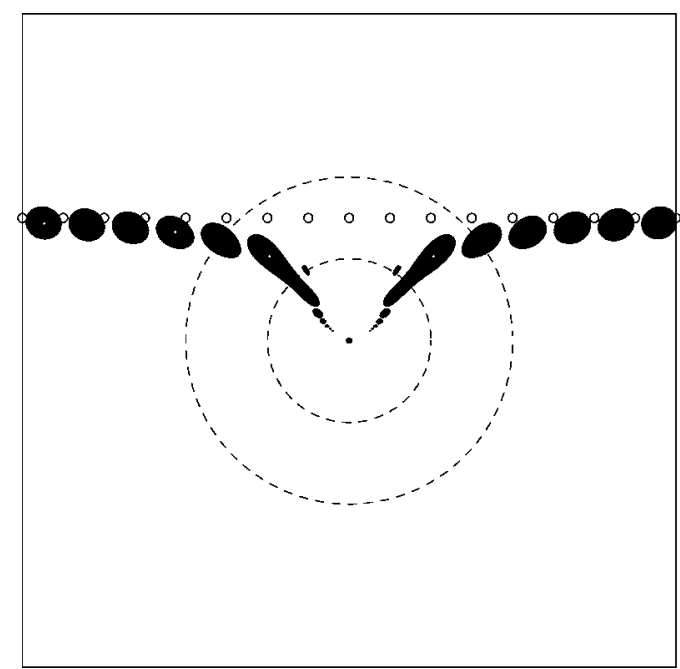

FIG. 8. True motion of the source and apparent motion of the images for $B_{0}<2$. The inner dashed circle is the Einstein ring, the outer dashed circle is twice the Einstein ring. The rest is as in Fig. 5.

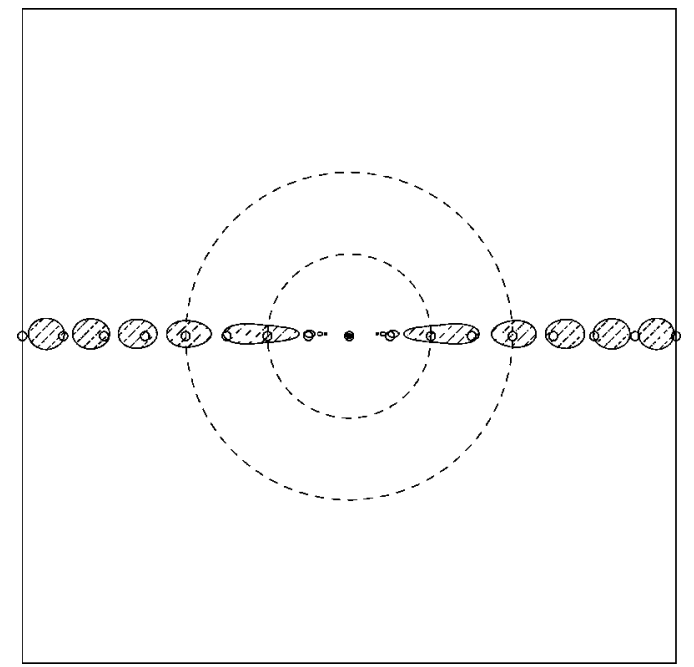

FIG. 9. True motion of the source and apparent motion of the images for $B_{0}=0$. The inner dashed circle is the Einstein ring, the outer dashed circle is twice the Einstein ring. Images here are shown with the shaded ellipses. The rest is as in Fig. 5.

$$
B_{0}=\frac{\beta_{0}}{\theta_{\mathrm{E}}},
$$

we obtain

$$
u(t)=\sqrt{B_{0}^{2}+\left(\frac{t}{t_{v}}\right)^{2}} .
$$

Finally, the total amplification as a function of time is given by

$$
A(t)=\frac{u(t)^{2}-2}{u(t) \sqrt{u(t)^{2}-4}} .
$$

Comparing this analysis with that of Cramer et al. [13], we must note that they wrote the equation for the time dependent dimensionless impact parameter as [cf. our Eq. (43)]

$$
B(t)=B_{0} \sqrt{1+\left(\frac{t}{T_{0}}\right)^{2}},
$$

and defined the time scale for the microlensing event as the time it takes to cross the minimum impact parameter [cf. our Eq. (39)]

$$
T_{0}=\frac{b_{0}}{V},
$$

where $b_{0}$ is the minimum impact parameter and other variables carry the same meanings as in our paper. While there is no mistake in using such definitions, there is a definite disadvantage in doing so. Using Eq. (10) of [13] for $B(t)$ we cannot build the light curve for the case of the minimum impact parameter $B_{0}=0$. In this case their Eq. (8) diverges, although there is nothing wrong with this value of $B_{0}$ (see our Figs. 9 and 10). In the same way, their definition of a time scale does not give much information on the light 


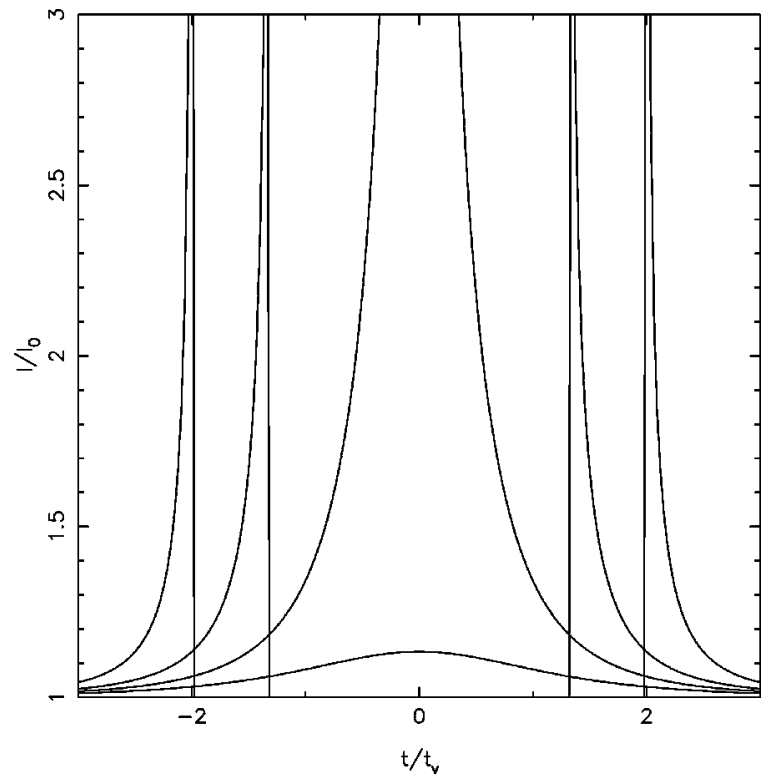

FIG. 10. Light curves for the negative mass lensing of a point source. From the center of the graph towards the corners the curves correspond to $B_{0}=2.5,2.0,1.5,0.0$. The time scale here is $\xi_{0}$ divided by the effective transverse velocity of the source.

curves. With our definition [Eq. (39)] we can see in Fig. 10 that in the extreme case of $B_{0}=0$ the separation between the half-events is exactly $2 \theta_{\mathrm{E}}$; it is always less than that with any other value of $B_{0}$.

In Fig. 10 we show the light curves for the point source for four source trajectories with different minimum impact parameters $B_{0}$. As can be seen from the light curves, when the distance from the point mass to the source trajectory is larger than $2 \theta_{\mathrm{E}}$, the light curve is identical to that of a positive mass lens light curve. However, when the distance is less than $2 \theta_{\mathrm{E}}$ (or in other terms, $B_{0} \leqslant 2.0$ ), the light curve shows significant differences. Such events are characterized by the asymmetrical light curves, which occur when a compact source crosses a critical curve. A very interesting, eclipse-like, phenomenon occurs here; a zero intensity region (disappearance of images) with an angular radius $\theta_{0}$

$$
\theta_{0}=\sqrt{4 \theta_{\mathrm{E}}^{2}-\beta_{0}^{2}},
$$

or in terms of normalized unit $\theta_{\mathrm{E}}$,

$$
\Delta=\sqrt{4-B_{0}^{2}} .
$$

In Sec. IV we shall see how these features get affected by the presence of an extended source.

\section{EXTENDED SOURCE}

In the previous sections we considered magnifications and light curves for point sources. However, sources are extended, and although their size may be small compared to the relevant length scales of a lensing event, this extension definitely has an impact on the light curves, as will be demonstrated below. From variability arguments, the optical and $\mathrm{x}$-ray continuum emitting regions of quasars are assumed to be much less than $1 \mathrm{pc}$ [23], whereas the broad-line emission probably has a radius as small as $0.1 \mathrm{pc}$ [24]. The high energy gamma-spheres have a typical radius of $10^{15} \mathrm{~cm}$ [25]. Hence, one has to consider a fairly broad range of source sizes.

We define here the dimensionless source radius, $\widetilde{R}$, as

$$
\widetilde{R}=\frac{\rho}{\theta_{\mathrm{E}}}=\frac{R}{\xi_{0}},
$$

where $\rho$ and $R$ is the angular and the linear physical size of the source, respectively, and $\xi_{0}$ is the the length unit in the source plane [see Eq. (38)].

\section{A. Comments on numerical method and simulations}

It is convenient to write the lens equation in the scaled scalar form

$$
y=x+\frac{1}{x}
$$

where we normalized the coordinates to the Einstein angle: ${ }^{1}$

$$
x=\frac{\theta}{\theta_{\mathrm{E}}} ; \quad y=\frac{\beta}{\theta_{\mathrm{E}}} .
$$

The lens equation can be solved analytically for any source position. The amplification factor, and thus the total amplification, can be readily calculated for point sources. However, as we are interested in extended sources, this amplification has to be integrated over the source [Eq. (50)], and furthermore, as we want to build the light curves, the total amplification for an extended source has to be calculated for many source positions. The amplification $\mathcal{A}$ of an extended source with surface brightness profile $I(\mathbf{y})$ is given by

$$
\mathcal{A}=\frac{\int d^{2} y I(\mathbf{y}) \mathcal{A}_{0}(\mathbf{y})}{\int d^{2} y I(\mathbf{y})}
$$

where $\mathcal{A}_{0}(\mathbf{y})$ is the amplification of a point source at position y. We have used the numerical method first described in [26]. We cover the lens plane with a uniform grid. Each pixel on this grid is mapped, using Eq. (48), into the source plane. The step width $(5000 \times 5000)$ is chosen according to the desired accuracy (i.e. the observable brightness). For a given source position $\left(y_{01}, y_{02}\right)$ we calculate the squared deviation function (SDF)

$$
S^{2}=\left(y_{10}-y_{1}\left(x_{1}, x_{2}\right)\right)^{2}+\left(y_{20}-y_{2}\left(x_{1}, x_{2}\right)\right)^{2} .
$$

The solutions of the lens equation (Eq. 49) are given by the zeroes of the SDF. Besides, Eq. (51) describes circles with

\footnotetext{
${ }^{1}$ Note, that for the case in which $x$ and $y$ are expressed in length units, we obtain a different normalization in each plane, which is not always convenient.
} 

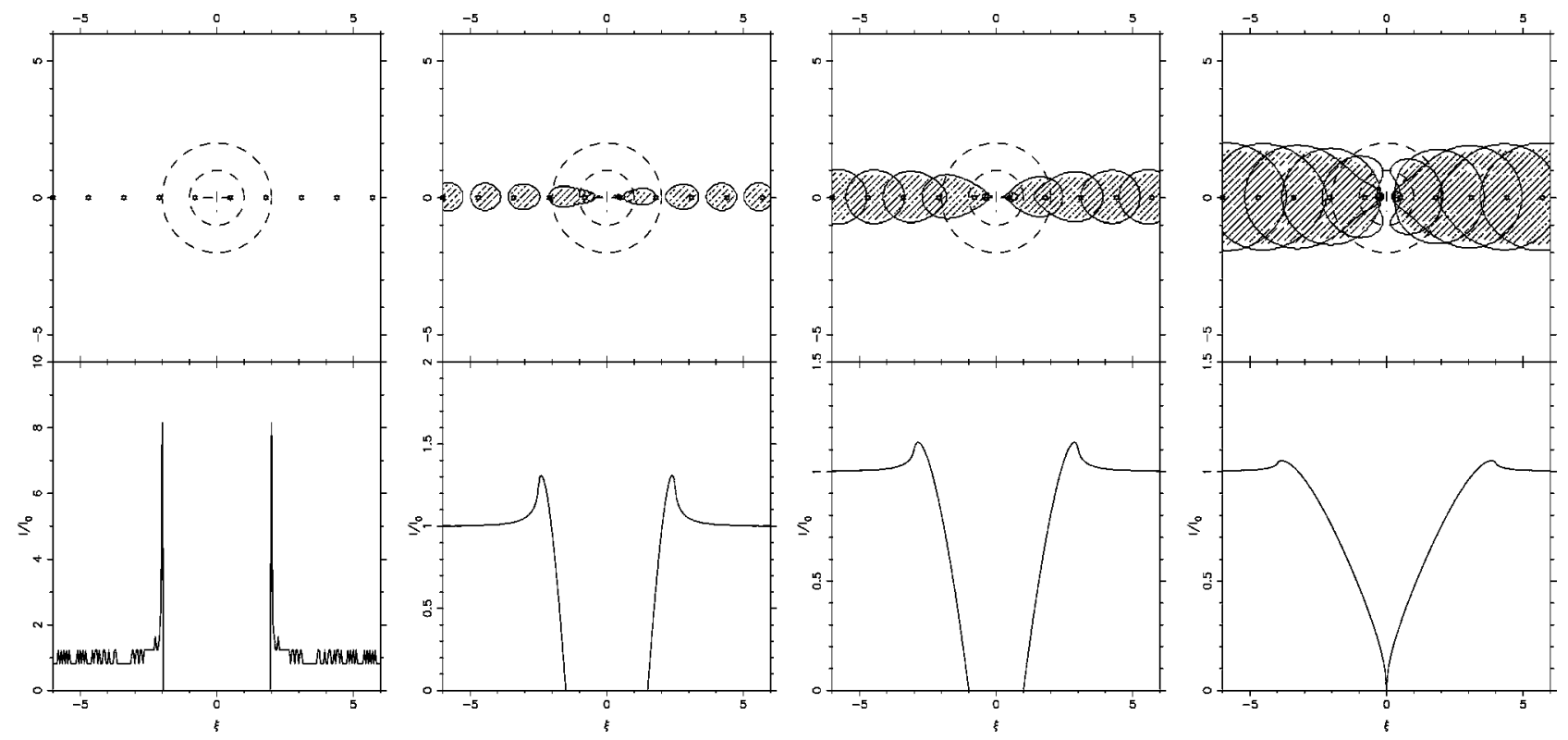

FIG. 11. Four sets of lens-source configurations (upper panels) and corresponding amplification as a function of the source's center position (bottom panels) are shown for four different values of the dimensionless source radius $\widetilde{R}(0.01,0.5,1.0,2.0$, in normalized units, $\left.\theta_{\mathrm{E}}\right)$. Each of the four upper panels display the time dependent position of the source's center, the shapes of images (shaded ellipses) and critical curves (dashed circles). The series of open small circles show the path of the source center. The lens is marked by the central cross. Minimum impact parameter $B_{0}=0$. By replacing $\xi$ with $\xi \theta_{\mathrm{E}} D_{\mathrm{s}} V^{-1}=\xi t_{\mathrm{v}}$ we get the corresponding time dependent light curve.

radii $S$ around $\left(y_{10}, y_{20}\right)$ in the source plane. Thus, the lines $S=$ const are just the image shapes of a source with radius $S$, which we plot using standard plotting software. Therefore, image points where SDF has value $S^{2}$ correspond to those points of the circular source which are at a distance $S$ from the center. The surface brightness is preserved along the ray and if $I\left(R_{0}\right)=I_{0}$ for the source, then the same intensity is given to those pixels where $\mathrm{SDF}=R_{0}^{2}$. In this way an intensity profile is created in the image plane and integrating over it we can obtain the total intensity of an image. Thus, we obtain the approximate value of the total magnification by estimating the total intensity of all the images and dividing it by that of the unlensed source, according to the corresponding brightness profile of the source (see Appendix B). For a source with the constant surface brightness the luminosity of the images is proportional to the area enclosed by the line $S=$ const. The total magnification is obtained by estimating the total area of all images and dividing it by that of the unlensed source. For calculations of light curves we used a circular source which is displaced along a straight line in the source plane with steps equal to 0.01 of the Einstein angular radius.

\section{B. Results}

In Figs. 11 and 12 we show four projected source and image positions, critical curves or caustics in the lens or source plane and representative light curves for different normalized source sizes. The sources are taken to be circular disks with constant surface brightness. In order to get absolute source radii and real light curves we need the value of $\theta_{\mathrm{E}}$, the normalized angular unit, the distance to the source, as well as the velocity $V$ of the source relative to the critical curves in the source plane (see Appendix A). We have used $M=M_{\odot}, H_{0}=100 \mathrm{~km} \mathrm{~s}^{-1} \mathrm{Mpc}^{-1}$ and a standard cosmological model with zero cosmological constant. Here and in all subsequent simulations the redshift of the source is $z_{\mathrm{s}}$ $=0.5$ and the redshift of the lens is $z_{1}=0.1$.

We display two cases for two different impact parameters. It must be noted that the minimum impact parameter $B_{0}$ now defines the shortest distance between the line of path of the center of the source and the lens. For each one of $B_{0}$, the dimensionless radius of the source $\widetilde{R}$ increases from 0.01 to 2.0 in normalized units of $\theta_{\mathrm{E}}$. The shape of produced images changes notably with the increase of the source size, as can be seen in the bottom right panel of Figs. 11 and 12. At the same time the smaller the source the greater the magnification, since when the source radius is greater than the Einstein radius of the lens, the exterior parts, which are amplified, compete with the interior ones, which are demagnified.

It can be noted that, despite the noise in some of the simulated light curves, the sharp peaks which occur when the source is crossing the critical line are well defined even for the smallest source. Note that all infinities are replaced now by finite amplifications, and that the curves are softened; all these effects are generated by the finite size of the source. Indeed, while the impressive drop to zero in the light curve is maintained, the divergence to infinity, that happens for a point source, is very much reduced. Note, that in cases of a large size of the source the magnification is very small. If we would like to see a bigger enhancement than that, we should consider sources of smaller sizes, approaching the point source situation (cf. Fig. 11, upper left plot).

It is also interesting to note that for the impact parameter $B_{0}=2.0$ the light curve of a small extended source, though 

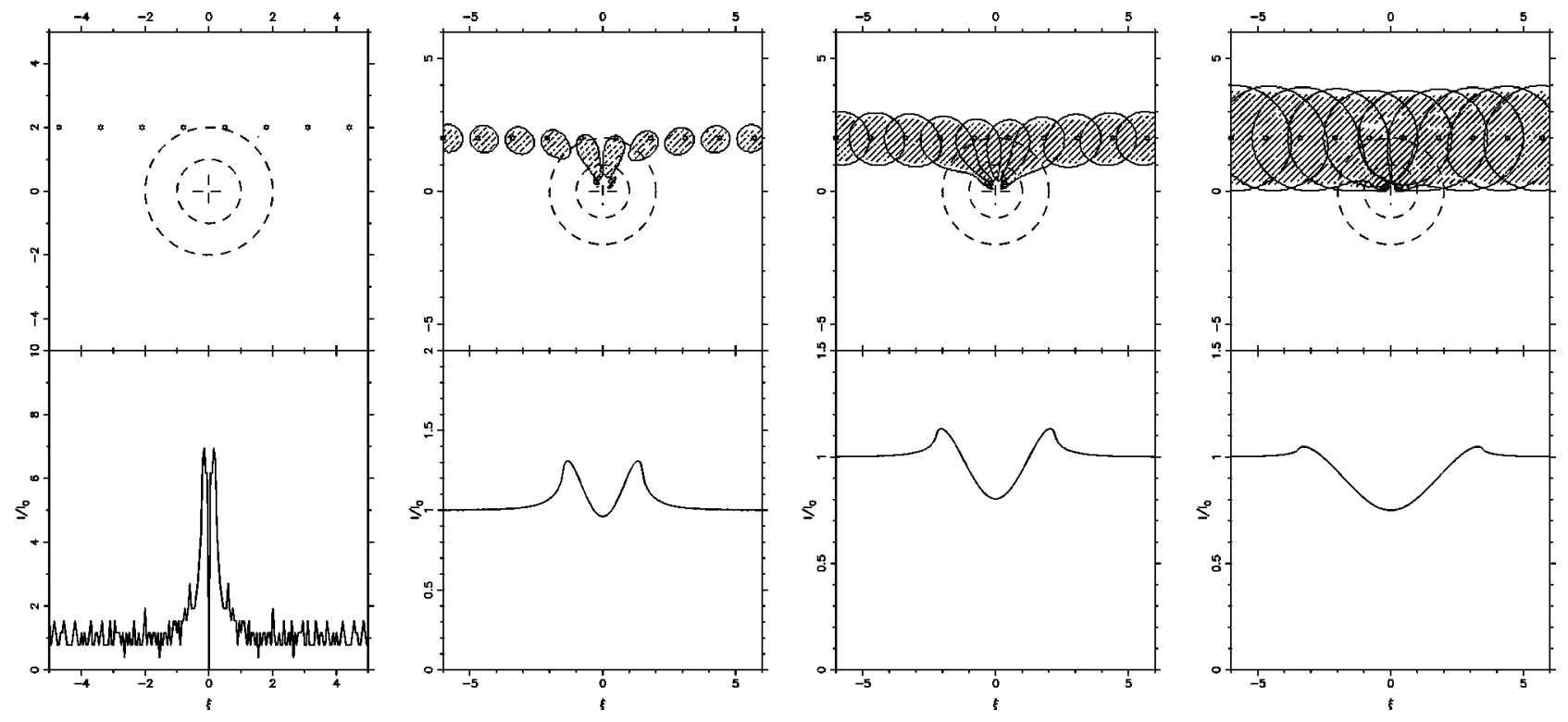

FIG. 12. Same as in Fig. 11, but for minimum impact parameter $B_{0}=2.0$.

approaching the point source pattern (Fig. 10), still differs considerably from it (Fig. 12, upper left plot).

In Figs. 13 and 14 we show the images of an extended source with a Gaussian brightness distribution for two effective dimensionless source radii (Appendix B), $\widetilde{R}_{\mathrm{S}}$, of 3.0 and 1.0 [Figs. 13(a)-13(e) and Figs. 14(a)-14(e), respectively], together with the corresponding light curves [Fig. 13(f) and Fig. 14(f), respectively]. Here the source path passes through the lens $\left(B_{0}=0\right)$, which lies exactly in the center of each frame. In Fig. 13 the source's extent in the lens plane is greater than the Einstein radius of the lens. There we notice that there is an eclipse-like phenomenon, occurring most notably when most of the source is near or exactly behind the lens. This is consistent with the light curve [Fig. 13(f)], where there is a de-magnification. For the source with radius smaller than the double Einstein radius of the lens (Fig. 14), the low intensity region is replaced by the zero intensity region; the source completely disappears from the view [Fig. 14(c)].

In Fig. 15 we display the images of the source with the exponential brightness profile (see Appendix B) and the corresponding light curve [Fig. 15(f)]. The effective radius of the source is 1.5 . The impact parameter is $B_{0}=2.0$; the lensing regime corresponds to the one schematically depicted in Fig. 7. We see how shapes of the images change, becoming elongated and forming the radial arc [Figs. 15(c) and 15(d)].

In order to compare a constant surface brightness source with more realistic distributions, we simulate images configurations and calculate light curves for two different assumed profiles with radial symmetry (see Appendix B).

In Fig. 16 we compare light curves for three different radially symmetric source profiles, uniform, Gaussian and exponential, for two dimensionless source radii $\widetilde{R}=\widetilde{R}_{\mathrm{S}}^{\text {gauss }}$ $=\widetilde{R}_{\mathrm{S}}^{\text {expon }}=0.1$ and $\widetilde{R}=\widetilde{R}_{\mathrm{S}}^{\text {gauss }}=\widetilde{R}_{\mathrm{S}}^{\text {expon }}=1.0$. As a reference curve we show the light curve of the point source. All curves are made for the impact parameter $B_{0}=0$. We can see the larger noise in the uniform source curve, since the source with uniform brightness has extremely sharp edge, whereas Gaussian and exponential sources are extremely smooth. Though we considered the sources with the same effective radius, we can see from the plot that for a small source size, the maximum magnification is reached by the source with exponential profile (upper panel), which is explained by the fact that this profile has a more narrow central peak than the Gaussian.

For the larger source, this behavior smoothens, though we still can see large differences in the light curves (bottom panel), where the uniform source experiences darkening, while sources with other profiles only undergo demagnification.

\section{CONCLUDING REMARKS}

In this paper we have explored the consequences, regarding gravitational microlensing, of the existence of matter violating the energy conditions. We have also quantitatively analyzed, using numerical simulations, the influence of a finite size of the source on the gravitational lensing negativemass event. We have thus enhanced and completed previous works, where the focus was put on the point source light curves and no discussion was given concerning the shapes of images, actual simulations of microlensing events, time gain function, and other features presented here. Figures 4, 6-9 and 11-16 comprise our new results: a useful comparison arena where the possible existence of wormholes or any other kind of negative mass compact objects can be tested observationally.

The next step would be to test these predictions using archival, current, and forthcoming observational microlensing experiments. The only search done up to now included the BATSE database of gamma-ray bursts, but there is still much unexplored territory in the gravitational microlensing archives. We suggest to adapt the alert systems of these experiments in order to include the possible effects of negative 
a)

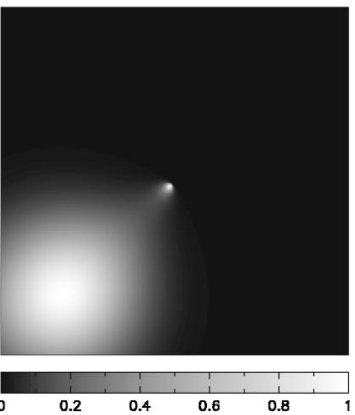

b)
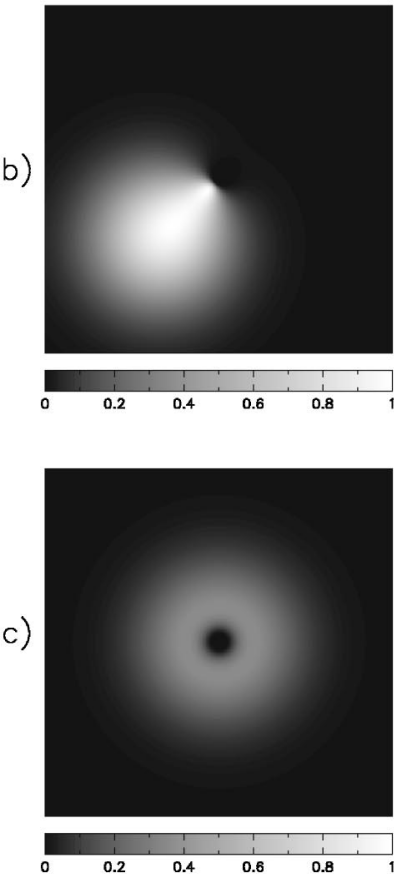

d)

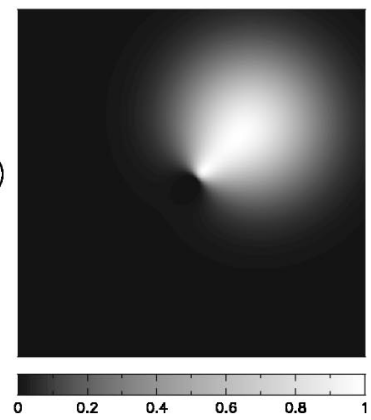

e)
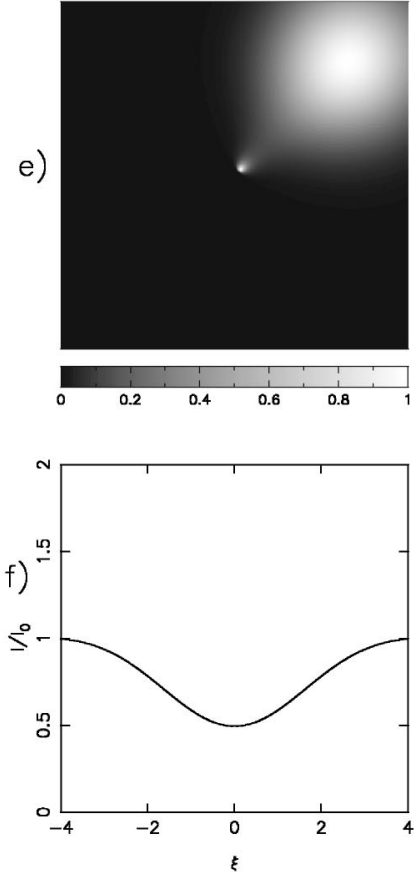

FIG. 13. Image configurations $[(\mathrm{a})-(\mathrm{e})]$ and a corresponding light curve (f) for a Gaussian source with effective radius $\widetilde{R}_{\mathrm{S}}=3.0$, in units of the Einstein angle. The source is moving from the lower left corner (a) to the right upper corner (e), passing through the lens $\left(B_{0}=0\right)$. The lens is in the center of each frame. Size of each frame is $5 \times 5$, in the normalized units. Wedges to each frame provide the brightness scale for the images. Note the eclipse-like phenomenon, consistent with the incomplete demagnification shown in the light curve (f).

masses as well. This, perhaps, would not imply a burdensome work, but there could be a whole new world of discoveries.

\section{ACKNOWLEDGMENTS}

M.S. is supported by a ICCR scholarship (Indo-Russian Exchange program) and acknowledges the hospitality of IUCAA, Pune. We would like to deeply thank Tarun Deep Saini for his invaluable help with the programming and Professor Daksh Lohiya and Professor M. V. Sazhin for useful discussions. This work was also supported by CONICET (PIP 0430/98, GER), ANPCT (PICT 98 No. 03-04881, GER) and Fundación Antorchas (through separate grants to DFT and

a)

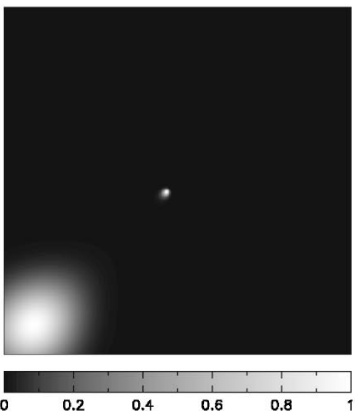

b)

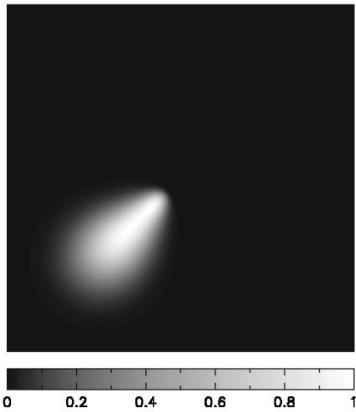

c)

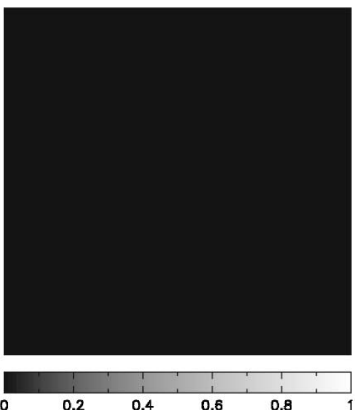

d)
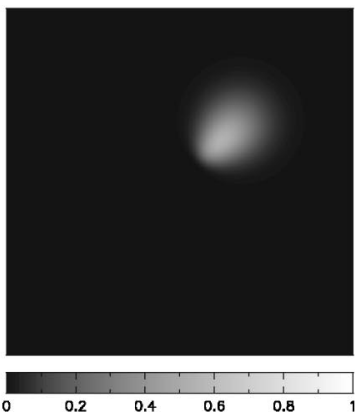

e)
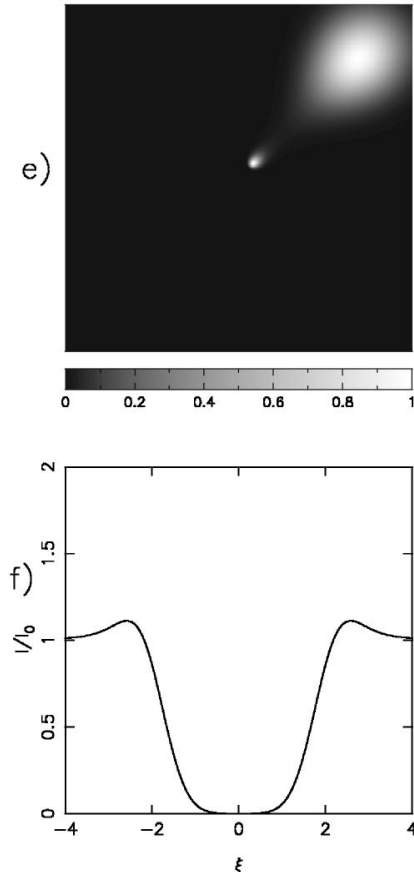

FIG. 14. Image configurations (a)-(e) and a corresponding light curve (f) for a Gaussian source with effective radius $\widetilde{R}_{\mathrm{S}}=1.0$, in units of the Einstein angle. The source is moving from the lower left corner (a) to the right upper corner (e), passing through the lens $\left(B_{0}=0\right)$. The lens is in the center of each frame. The size of each frame is $3 \times 3$, in the normalized units. Wedges to each frame provide the brightness scale for the images. Note the complete disappearence of the source when it is inside the double Einstein radius of the lens (c), corresponding to the drop of magnification to zero in the light curve (f).

GER). D.F.T. especially acknowledges I. Andruchow for her continuing support.

\section{APPENDIX A: VELOCITIES AND TIME SCALES}

Let the source have a transverse velocity $\mathbf{v}_{\mathrm{S}}$ measured in the source plane, the lens a transverse velocity $\mathbf{v}_{1}$ measured in the lens plane, and the observer a transverse velocity $\mathbf{v}_{\text {obs }}$ measured in the observer plane. The effective transverse velocity of the source relative to the critical curves with time measured by the observer is

$$
\mathbf{V}=\frac{1}{1+z_{\mathrm{s}}} \mathbf{v}_{\mathrm{s}}-\frac{1}{1+z_{1}} \frac{D_{\mathrm{s}}}{D_{1}} \mathbf{v}_{1}+\frac{1}{1+z_{1}} \frac{D_{\mathrm{ls}}}{D_{1}} \mathbf{v}_{\mathrm{obs}},
$$


a)
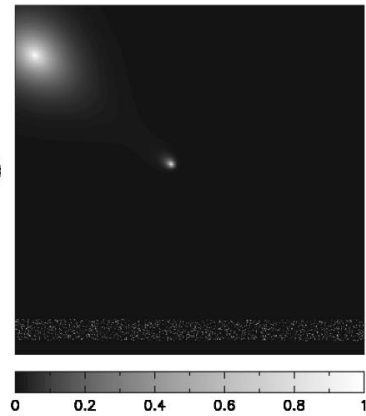

b)

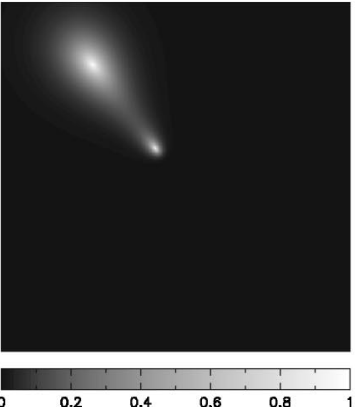

c)

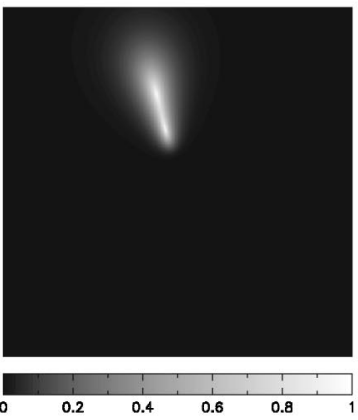

d)

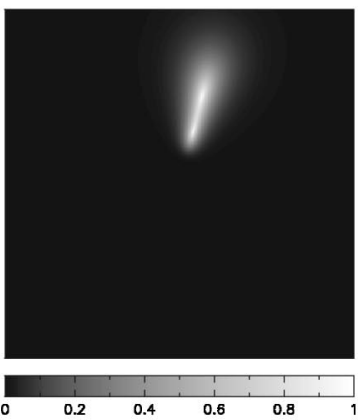

e)
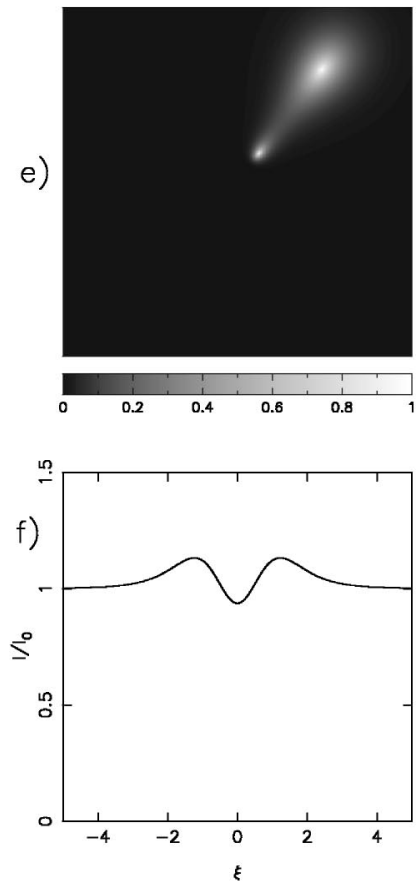

FIG. 15. Image configurations $[(\mathrm{a})-(\mathrm{e})]$ and a corresponding light curve (f) for a source with the exponential brightness distribution and effective radius $\widetilde{R}_{\mathrm{S}}=1.0$, in units of the Einstein angle. The source is moving from the upper left corner (a) to the upper right corner (e) with the impact parameter $B_{0}=2.0$. The lens is in the center of each frame. Size of each frame is $2.5 \times 2.5$, in the normalized units. Wedges to each frame provide the brightness scale for the images.

where this effective velocity is such that for a stationary observer and lens, the position of the source will change in time according to $\delta \boldsymbol{\xi}=\mathbf{V} \Delta t$.

We basically have two time scales of interest here. The first one is the typical rise time to a peak in the amplification. We can estimate that it corresponds to a displacement of $\Delta y \sim \widetilde{R}$ of the source across a critical line; the corresponding time scale $\tau_{1}$ is

$$
\tau_{1}=t_{\mathrm{v}} \widetilde{R}
$$

where $t_{\mathrm{v}}$ is given by Eq. (39), or in terms of the physical source size $R=\xi_{0} \widetilde{R}$,

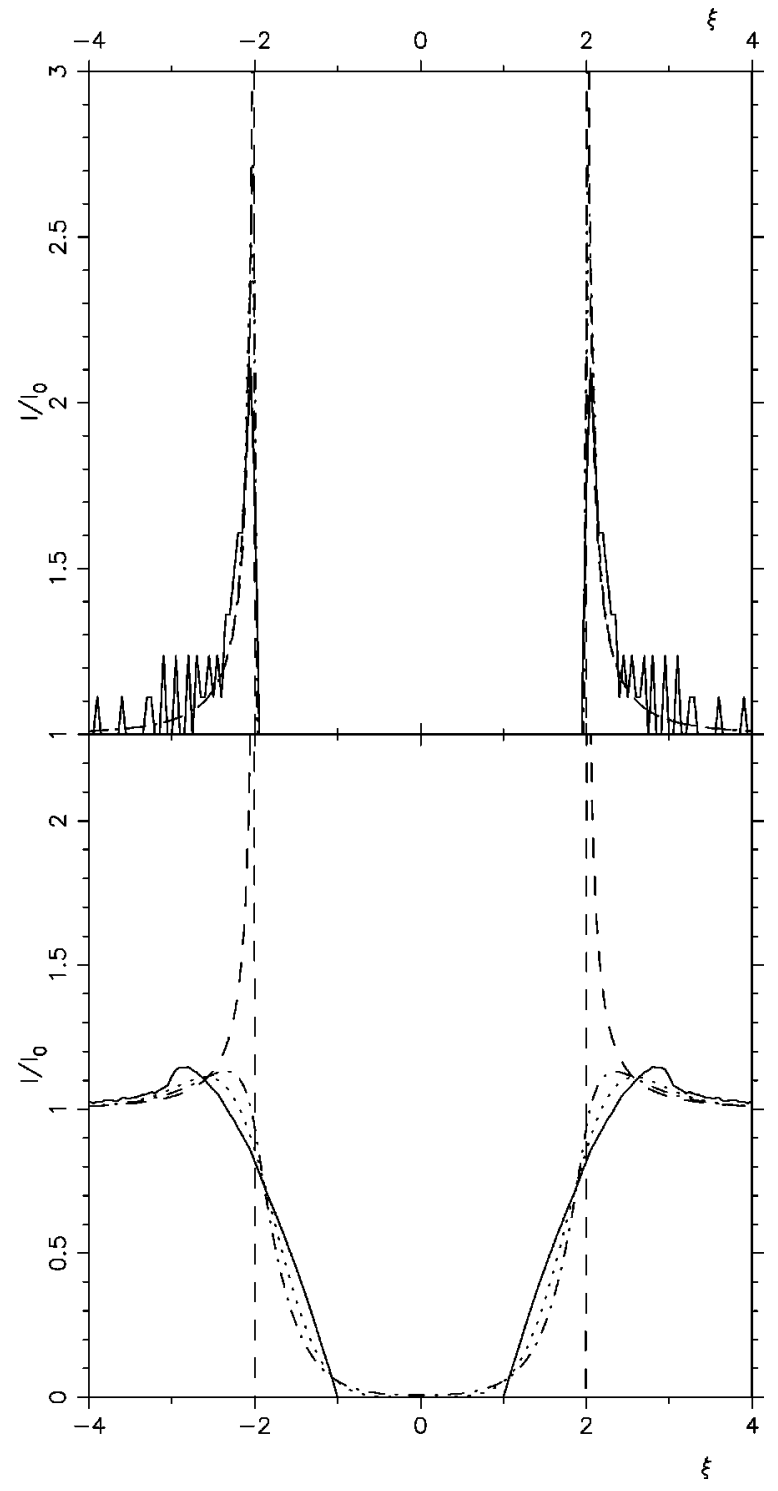

FIG. 16. Light curves for the point mass source (dashed line), source with constant surface brightness (solid), source with Gaussian brightness distribution (dash-dotted) and exponential brightness distribution (dotted) for two different effective dimensionless source radii, 0.1 (upper panel) and 1.0 (bottom panel).

$$
\tau_{1}=\frac{R}{V},
$$

where effective transverse velocity of the source $V$ is given by Eq. (A1). The second time scale of interest is the time between two peaks $\tau_{2}$. For a point source we can estimate it as

$$
\tau_{2}=t_{\mathrm{v}} \Delta
$$

where $\Delta$ is given in Eq. (46). For a source with radius $\widetilde{R}$ and impact parameter $B_{0}=0.0$ it can be shown to be

$$
\tau_{2}=t_{\mathrm{v}}(\Delta+\widetilde{R})
$$


TABLE I. Time scales for several source radii. $\widetilde{R}$ is the dimensionless source radius, in units of Einstein angle, $|M|=1.0 M_{\odot}$, redshift of the lens is $z_{1}=0.1$, redshift of the source is $z_{\mathrm{s}}=0.5, \xi_{0}$ $=5.42 \times 10^{11} \mathrm{~km}$ is the normalized length unit in the source plane. The time scales correspond to apparent source velocity [see Eq. (A1)] $V=5000 \mathrm{~km} \mathrm{~s}^{-1}$.

\begin{tabular}{lcccc}
\hline \hline$\widetilde{R}$ & $R(\mathrm{pc})$ & $\Delta t_{12}{ }^{\mathrm{a}}(\mathrm{sec})$ & $\tau_{1}(\mathrm{yr})$ & $\tau_{2}{ }^{\mathrm{b}}(\mathrm{yr})$ \\
\hline 0.0 & point source & $2.0 \times 10^{-4}$ & & 6.78 \\
0.01 & $1.07 \times 10^{-4}$ & & 0.03 & 6.81 \\
0.1 & $1.07 \times 10^{-3}$ & & 0.34 & 7.11 \\
1.0 & $1.07 \times 10^{-2}$ & & 3.38 & 10.16 \\
2.0 & $0.3 \times 10^{-1}$ & & 6.75 & 13.6 \\
\hline \hline
\end{tabular}

${ }^{\mathrm{a}} \mathrm{u}=4.0$ [definition in Eq. (29)].

${ }^{\mathrm{b}} B_{0}=0.0$.

In Table I we list for different values of source radii the time scales $\tau_{1}$ and $\tau_{2}$, and the time delay between two images $\Delta t_{12}$ for the point source. We estimate the value of $V$ to be $V=5000 \mathrm{~km} \mathrm{~s}^{-1}$.

\section{APPENDIX B: EXTENDED SOURCES BRIGHTNESS PROFILES}

\section{Source of uniform brightness}

For a circular source of radius $R$ and uniform brightness, Eq. (49) transforms into

$$
\mathcal{A}=\frac{\int d^{2} y I(\mathbf{y}) \mathcal{A}_{0}(\mathbf{y})}{\pi R^{2}} .
$$

\section{Source with Gaussian brightness distribution}

For a Gaussian source we have

$$
I(r)=I_{0} e^{-r^{2} / r_{0}^{2}},
$$

where we normalized the profile such that the maximum value of $I$ equals unity. We define the radius containing $90 \%$ of all the luminosity as the effective radius of the source, $R_{\mathrm{S}}$. To find the relation between $R_{\mathrm{S}}$ and $r_{0}$, we write the total luminosity as

$$
L(\infty)=\int_{0}^{\infty} e^{-r^{2} / r_{0}^{2}} 2 \pi r d r=\pi r_{0}^{2}
$$

$$
L(R)=\int_{0}^{R} e^{-r^{2} / r_{0}^{2}} 2 \pi r d r=\pi r_{0}^{2}\left[1-e^{-R^{2} / r_{0}^{2}}\right]
$$

then

$$
\frac{L\left(R_{\mathrm{S}}\right)}{L(\infty)}=0.9=\left[1-e^{-R_{\mathrm{S}}^{2} / r_{0}^{2}}\right]
$$

Thus, the effective radius relates to the parameter $r_{0}$ as

$$
\frac{R_{\mathrm{S}}}{r_{0}}=\sqrt{\ln 10}
$$

\section{Source with exponential brightness distribution}

We have

$$
I(r)=I_{0} e^{-r / r_{0}}
$$

In the same manner as above, $R_{\mathrm{S}}$ is defined as radius containing $90 \%$ of total luminosity. In the same way as above, total luminosity

$$
L(\infty)=\int_{0}^{\infty} e^{-r / r_{0}} 2 \pi r d r=2 \pi r_{0}^{2}
$$

then

$$
L(R)=\int_{0}^{R} e^{-r / r_{0}} 2 \pi r d r=2 \pi\left[r_{0}^{2}-\left(R r_{0}+r_{0}^{2}\right) e^{-R / r_{0}}\right]
$$

and

$$
\frac{L\left(R_{\mathrm{S}}\right)}{L(\infty)}=0.9
$$

where we find that effective radius relates to the parameter $r_{0}$ as

$$
e^{-R_{\mathrm{S}} / r_{0}\left(1+\frac{R_{\mathrm{S}}}{r_{0}}\right)}=0.1
$$

The solution to this equation gives $R_{\mathrm{S}} / r_{0} \approx 3.89$. This profile is also normalized such that the maximum value of $I$ equals unity.
[1] M.S. Morris and K.S. Thorne, Am. J. Phys. 56, 395 (1988).

[2] L.A. Anchordoqui, S.E. Perez Bergliaffa, and D.F. Torres, Phys. Rev. D 55, 5226 (1997); A. DeBenedictis and A. Das, Class. Quantum Grav. 18, 1187 (2001); S.E. Perez Bergliaffa and K.E. Hibberd, Phys. Rev. D 62, 044045 (2000); C. Barcelo and M. Visser, Nucl. Phys. B584, 415 (2000); L.A. Anchordoqui and S.E. Perez Bergliaffa, Phys. Rev. D 62, 067502 (2000); D. Hochberg, A. Popov, and S.V. Sushkov, Phys. Rev.
Lett. 78, 2050 (1997); S. Kim and H. Lee, Phys. Lett. B 458, 245 (1999); S. Krasnikov, Phys. Rev. D 62, 084028 (2000); D. Hochberg and M. Visser, ibid. 56, 4745 (1997).

[3] M. Visser, Lorentzian Wormholes (AIP, New York, 1996).

[4] D. Hochberg and M. Visser, Phys. Rev. Lett. 81, 746 (1998).

[5] D. Hochberg and M. Visser, Phys. Rev. D 58, 044021 (1998).

[6] C. Barceló and M. Visser, Phys. Lett. B 466, 127 (1999); M. Visser, talk at the Marcel Grossman Meeting, Rome, 2000; C. 
Barcelo and M. Visser, Class. Quantum Grav. 17, 3843 (2000).

[7] M. Jammer, Concepts of Mass (Dover, New York, 1997).

[8] H. Bondi, Rev. Mod. Phys. 29, 423 (1957).

[9] M. Visser, Phys. Rev. D 39, 3182 (1989); Nucl. Phys. B328, 203 (1989).

[10] T. Roman, Phys. Rev. D 47, 1370 (1993).

[11] R.B. Mann, Class. Quantum Grav. 14, 2927 (1997).

[12] S.-W. Kim and Y.M. Cho, in Evolution of the Universe and its Observational Quest (Universal Academy Press, Inc. and Yamada Science Foundation, 1994), p. 353.

[13] J. Cramer, R. Forward, M. Morris, M. Visser, G. Benford, and G. Landis, Phys. Rev. D 51, 3117 (1995).

[14] D.F. Torres, G.E. Romero, and L.A. Anchordoqui, Phys. Rev. D 58, 123001 (1998); Mod. Phys. Lett. A 13, 1575 (1998).

[15] L. Anchordoqui, G.E. Romero, D.F. Torres, and I. Andruchow, Mod. Phys. Lett. A 14, 791 (1999).

[16] G.E. Romero, D.F. Torres, I. Andruchow, L.A. Anchordoqui, and B. Link, Mon. Not. R. Astron. Soc. 308, 799 (1999).

[17] S.F. Portegies Zwart, C-H. Lee, and H.K. Lee, Astrophys. J. 520, 666 (1999).
[18] M. Safonova, D.F. Torres, and G.E. Romero, Mod. Phys. Lett. A 16, 153 (2001).

[19] E. Eiroa, G.E. Romero, and D.F. Torres, Mod. Phys. Lett. A 16, 973 (2001).

[20] P. Schneider, J. Ehlers, and E.E. Falco, Gravitational Lenses (Springer-Verlag, Berlin, 1992).

[21] R. Narayan and M. Bartelmann, in Formation of Structure in the Universe, edited by A. Dekel and J.P. Ostriker (Cambridge University Press, Cambridge, England, 1999), p. 360.

[22] R. Kayser, S. Refsdal, and R. Stabell, Astron. Astrophys. 166, 36 (1986).

[23] P. Schneider and A. Weiss, Astron. Astrophys. 171, 49 (1987).

[24] D.E. Osterbrock, A.T. Koski, and M.M. Phillips, Astrophys. J. 206, 898 (1976).

[25] R.D. Blanford and A. Levinson, Astrophys. J. 441, 79 (1995).

[26] T. Schramm and R. Kayser, Astron. Astrophys. 174, 361 (1987).

[27] M.V. Sazhin and A.F. Zakharov, Usp. Fiz. Nauk 168, 1041 (1998). 